\title{
Gestión de riesgo de desastres en instituciones de educación superior : una mirada desde la aplicación de un proyecto
}

\section{Disaster risk management in higher education institutions, a view from a project application}

\begin{abstract}
María Victoria Zavala Saucedo ${ }^{1 *}{ }^{\bullet}$, Karen Natali Backes Dos Santos ${ }^{1}{ }^{\oplus}$, Silvia Mercedes Ríos Zavala $^{2}{ }^{\bullet}$, Diana Stella Colmán ${ }^{\mathbf{1}, \mathbf{2}}{ }^{1}$ Universidad Nacional del Este. Escuela de Posgrado. Ciudad del Este, Paraguay ${ }^{2}$ Instituto Nacional de Educación Superior Dr. Raúl Peña. Asunción, Paraguay *Autor de correspondencia: mavizavala@gmail.com.
\end{abstract}

Recibido: 2 mayo 2021

Aceptado: 29 junio 2021

Recibido en versión modificada: 29 junio 2021

Zavala Saucedo, M. V., Backes Dos Santos, K. N., Ríos Zavala, S. M. \& Colmán, D. S. Gestión del riesgo de desastres en instituciones de educación superior, una mirada desde la aplicación de un proyecto. Investigaciones y Estudios - UNA, 12(1), 58-63.

https://doi.org/10.47133/IEUNA2116

Resumen. La gestión y reducción de riesgo de desastres se plantea como desafío para las universidades, por ello este artículo describió la experiencia en la gestión de un proyecto identificando las actividades y analizando los resultados, para lo cual se recurrió a documentos relacionados con él disponibles en la web. Se identificaron las etapas de ejecución con sus actividades y resultados. El desarrollo del curso taller favoreció la apropiación del marco conceptual y operativo de la temática, lo que permitió identificar las posibilidades de incorporación de la gestión del riesgo en actividades de enseñanza, extensión e investigación. Resaltan como buenas prácticas: los talleres sobre temas relacionados con la gestión de riesgos de desastres, la evaluación de la seguridad de la institución, la convocatoria a actores de diferentes instituciones en las actividades de capacitación y la inclusión en el proyecto de acciones que afectan a la formación e investigación.

Palabras clave. gestión de riesgo de desastres, buenas prácticas, rol de la universidad, gestión educativa.

Abstract. Disaster risk management and reduction is a challenge for universities. Thus, this article describes the experience in the management of a project by identifying the activities and analyzing the results, using related documents available on the web. The stages of execution with their activities and results were identified. The development of the workshop course favored the appropriation of the conceptual and operative framework of the subject, which made it possible to identify the possibilities of incorporating risk management in teaching, extension and research activities. The following were identified as good practices: the workshops on topics related to disaster risk management, the evaluation of the institution's safety, the summoning of actors from 
different institutions in training activities and the inclusion of actions that affect training and research.

Keywords. disaster risk management, higher education, best practices, educational management.

\section{INTRODUCCIÓN}

La gestión del riesgo de desastres constituye una temática compleja que puede ser entendida desde múltiples perspectivas y abordajes teóricometodológicos, más aún cuando se refiere a las líneas de acción y estrategias para su implementación en las organizaciones. Es compleja porque existen diferentes concepciones del riesgo que varían según épocas históricas, condiciones sociales y lugares donde se generan los modelos teóricos, como intentos de explicación y comprensión de una realidad producto del imaginario colectivo (Reyes-Rivero et al., 2017). Por otra parte, la gestión del riesgo como objeto de estudio se trata de un cuerpo teórico en proceso de construcción que ha evolucionado a través del tiempo (Peraldo \& Acevedo, 2014).

La evolución histórica de este concepto nos lleva a la definición de la gestión del riesgo como un proceso social orientado a "la previsión, reducción y control permanente de los factores de riesgo en la sociedad, integrada al logro de pautas de desarrollo humano, económico, ambiental y territorial, sostenibles" (p. 33). De esta manera, la gestión del riesgo comprende diferentes formas de intervención: formulación e implementación de políticas y estrategias, ejecución de acciones y creación de instrumentos concretos de reducción y control (Narváez et al., 2009).

Reyes Rivero et al. (2017), destacan el papel fundamental de la educación en el logro de sociedades más seguras, pues refieren que al ser el riesgo un proceso socialmente construido puede ser manejado, modificado y transformado.

También el Marco de Sendai para la Reducción del Riesgo de Desastres 2015-2030, al referirse a los desafíos futuros que dejó el Marco de Acción de Hyogo menciona la necesidad de colaboración entre los gobiernos, las organizaciones de la sociedad civil y la comunidad académica para desarrollar un enfoque preventivo del riesgo de desastres más amplio y centrado en las personas (UNISDR, 2015).

Así también, en la Prioridad 1, Comprender el riesgo, del Marco de Sendai se establece la relevancia de promover la incorporación de los conocimientos sobre el riesgo de desastres en todos los niveles y modalidades de la educación.

Los Objetivos de Desarrollo Sostenible (ODS) 2015 y el Marco de Sendai para la Reducción del Riesgo de Desastres 2015-2030 instan a que las organizaciones del sector público y privado, de la sociedad civil, así como la comunidad académica implementen acciones para la integración de estas agendas en sus marcos estratégicos y con ello contribuir al logro de un mundo resiliente $y$ seguro. Es así que Cosme Casulo (2018) destaca el papel relevante de la universidad en la consecución de los ODS, en lo referente a la producción de conocimientos y la vinculación social.

En este contexto la Red Universitaria de las Américas y el Caribe para la Reducción de Riesgo de Desastres (REDULAC/RRD) convocó a las universidades de Latinoamérica y el Caribe a presentar proyectos con acciones orientadas al desarrollo de una cultura institucional de gestión de riesgo de desastres, como una de las estrategias de la campaña "Universidades Sostenibles y Resilientes", durante el período 2018-2019. Esta campaña surge en el marco del Curso de Formación de Formadores para la elaboración de Planes de Reducción del Riego de 
Desastres con base en el Marco de Sendai, desarrollado en la ciudad de Panamá en el año 2016.

Este curso permitió visualizar la necesidad de atender con un instrumento específico al sector de la educación superior, tomando en cuenta las 11.000 instituciones existentes y la población de 40 millones de estudiantes (REDULAC, 2018).

La Campaña Universidades Sostenibles y Resilientes tuvo como propósito garantizar que las ciudades universitarias sean resilientes para desarrollar el proceso educativo en condiciones de seguridad y dar continuidad a sus servicios antes, durante y después de un desastre.

Este trabajo tiene como objetivo reportar la experiencia en el proceso de aplicación de un proyecto de Gestión de Riesgo de Desastres en una Institución de Educación Superior, con el fin de ilustrar una buena práctica posible de replicar en otras instituciones. Para responder a este objetivo: se describe cómo se ejecutó el proyecto en sus etapas y actividades y se analizan los resultados más resaltantes de la gestión del proyecto.

La experiencia objeto de análisis se desarrolló durante los años 2018 y 2019 en una IES del Paraguay cuyo proyecto fue seleccionado por REDULAC/RRD para su implementación con financiamiento de la Oficina Regional del Gobierno de los Estados Unidos de Asistencia para Desastres en el Exterior (OFDA) y la administración del Consejo Superior Universitario Centroamericano (CSUCA).

Para la elaboración de este reporte se realizó una revisión de documentos relacionados con el proyecto "Hacia una institución segura y resiliente", disponibles en la página de la institución beneficiada, además de otros textos de la Campaña "Universidades sostenibles y resilientes" de REDULAC/RRD.

\section{DESARROLLO}

A continuación, se describen cada una de las etapas y las actividades ejecutadas:

1. Etapa 1, año 2018

1.1. Taller de inducción al Proyecto

Esta actividad se desarrolló con el objetivo de promover la campaña Universidades Sostenibles y Resilientes y capacitar a actores claves de las IES en los temas centrales de dicha campaña. La Tabla 1 ilustra el perfil de los participantes del taller.

Tabla 1. Actores participantes y funciones.

\begin{aligned} \hline Actores & \multicolumn{1}{c}{ Funciones } \\ Gestión de Riesgo & IES, además de asesorar en la temática a las autoridades institucionales. \\ Técnico de la Unidad de & Gestionar acciones relacionadas a la reducción de riesgos con estudiantes y docentes \\ Gestión de Riesgo & de la IES. \\ Coordinador de Práctica & Vincular a las IES con las instituciones del Sistema Educativo Nacional en las que \\ Profesional & se realizan las actividades de práctica de los estudiantes. \\ Docente investigador & Desarrollar acciones de investigación en vinculación con las carreras. \\ Docentes & $\begin{array}{l}\text { Desarrollar actividades de enseñanza, investigación y extensión en las carreras } \\ \text { ofrecidas por las IES, según perfil profesional y área de formación. }\end{array} \\$ Técnicos de IES & $\begin{array}{l}\text { Planificar, implementar, monitorear y evaluar proyectos y programas educativos en } \\ \text { las IES. }\end{array} \\ &$ Especialista en elaboración de mapas y planos. \end{aligned}


Continúa Tabla 1

\begin{tabular}{c} 
Continúa Tabla 1 \\
\hline Actores \\
\hline $\begin{array}{c}\text { Jefe de capacitación en } \\
\text { institución del Estado }\end{array}$ \\
$\begin{array}{c}\text { responsable de la gestión de } \\
\text { riesgos de desastres en el país }\end{array}$ \\
\hline Fuente: elaboración propia a partir del Registro de Participantes.
\end{tabular}

El taller contó con la participación de docentes, técnicos, estudiantes de la institución coordinadora del proyecto y de representantes de instituciones de educación superior nacionales y privadas de la capital del país, del departamento Central, Itapúa y Alto Paraná, miembros de REDULAC/RRD, además de referentes de organizaciones del Estado y organismos de primera respuesta. Como resultado se puede destacar la participación de 46 actores institucionales capacitados en la elaboración de un Plan Institucional de Gestión de Riesgos y en el uso de la herramienta Índice de Seguridad de Instalaciones Universitarias (ISIU).

\subsection{Diagnóstico del entorno institucional.}

Se realizó un estudio acerca de la caracterización de los componentes de la institución, su ubicación geográfica y su entorno, de modo a recabar datos que permitan un diagnóstico de los riesgos, amenazas, vulnerabilidad, exposición. Estos hallazgos fueron sistematizados en: un mapa de georreferenciación, en el que se representa el posicionamiento espacial de la institución por medio de un sistema de coordenadas y puntos de referencia en la superficie terrestre; y un inventario arbóreo con una lista ordenada, georreferenciada, que describe las características de cada árbol ubicado en el Campus (especie, tamaño, circunferencia del tronco, altura, estado fitosanitario, afectaciones de cada árbol a la infraestructura).

En la evaluación de riesgos el mapa de arborización permitió la cuantificación de las especies arbóreas de tal manera a facilitar el mantenimiento y el monitoreo del arbolado del campus, así como disminuir los daños causados por la caída de los árboles mediante podas preventivas (INAES, 2019).

Para la concreción del diagnóstico se contó con el apoyo técnico del Instituto Geográfico Militar desarrollado en el Marco del Convenio de Incorporación y Cooperación entre el Comando de las Fuerzas Militares y la Red Universitaria de las Américas y el Caribe para la Reducción de Riesgo de Desastres Capítulo Paraguay (REDULAC/RRD Cap. Py) de fecha 9 de octubre del año 2018, cooperación que fue posible gracias a que el INAES forma parte de la misma red.

\subsection{Aplicación del Índice de Seguridad de}

Instalaciones Universitarias (ISIU).

Se realizó el análisis en dos edificios (bloques) del campus: la biblioteca y el laboratorio de Ciencias Básicas que se reportó en un informe técnico sobre las condiciones de la infraestructura evaluada.

El ISIU es un instrumento para identificar el nivel de seguridad ante desastres en las edificaciones de las instituciones de educación superior. Evalúa el sitio de emplazamiento, aspectos estructurales, aspectos no estructurales y la organización de una institución ante una emergencia (aspectos funcionales) (GarcíaLemus et al., 2017).

Por las características de la herramienta, cuya aplicación requiere de conocimientos especializados en aspectos estructurales (relacionados con la seguridad del edificio: tipo de material utilizado, 
estado de la edificación, irregularidades en planta, rigidez, masa y resistencia entre otros); no estructurales (líneas vitales, mobiliario y equipo, elementos arquitectónicos y otros) y funcionales (comité de emergencia, planes de respuesta, protocolos y otros) además de las condiciones requeridas en las especificaciones de la convocatoria del proyecto, fue necesario contar con profesionales ingenieros y arquitectos.

\section{Etapa 2, año 2019}

2.1. Taller Nacional sobre análisis de riesgos en Instituciones de Educación Superior.

Esta actividad estuvo dirigida a diferentes actores de IES miembros de REDULAC/RRD - Capítulo Paraguay, organizaciones gubernamentales (cuyas funciones están especificadas en la Tabla 1), contó con 40 participantes y se desarrolló durante 3 días en el INAES, el objetivo de esta fue compartir información referida a cómo identificar amenazas y vulnerabilidades en las IES y entorno.

2.2. Ampliación del diagnóstico de riesgos, amenazas y vulnerabilidades de la institución y su entorno.

Los resultados de este estudio fueron organizados en mapas: de vulnerabilidades, de recursos geológicos, de servicios ecosistémicos.

2.3. Diagnóstico de las condiciones de la infraestructura ampliado a dos edificios del campus.

Con el propósito de conocer las condiciones de seguridad de otras edificaciones de la institución (distintas a las de la etapa 1, año 2018) se aplicó el ISIU al salón auditorio y al Bloque donde se encuentran las oficinas administrativas.

2.4. Estudio sobre la inclusión de la Gestión de Riesgo de Desastres en los proyectos educativos de las diferentes carreras y programas del INAES.

Con el objetivo de identificar la presencia de temas vinculados a la Gestión de Riesgo de Desastres en los documentos curriculares, se conformó un equipo integrado por estudiantes y docentes liderado por la dirección de investigación. Las conclusiones de este estudio no fueron mencionadas en el informe final del Proyecto.

\section{RESULTADOS}

Para la identificación de resultados se recurrió al cuestionario aplicado a los participantes de las acciones de capacitación, a partir del cual se pudo recoger buenas prácticas para la incorporación de la gestión de riesgo de desastres en una institución de educación superior, tales como el desarrollo del curso taller para que los participantes se apropien del marco conceptual y operativo, lo cual según la consulta redundó en la identificación de posibilidades para la incorporación de la gestión de riesgos en las actividades docentes, de extensión e investigación y en el reconocimiento de estrategias para el análisis de escenarios de riesgo en las IES. También, a partir de la participación en los talleres los actores manifestaron haber emprendido actividades como: jornadas para estudiantes de diferentes carreras, promoción del voluntariado y acciones de extensión relacionados con la GRRD, conformación de brigadas de seguridad institucional, programas de prevención de riesgos laborales, capacitación para incorporar la gestión de riesgos en la agenda institucional, fortalecimiento de la gestión de riesgos en la Práctica Profesional a través de la elaboración de planes institucionales para las escuelas y colegios de pasantía, análisis de la situación institucional en torno a la gestión de riesgos. 
Por otra parte, el hecho de que en los dos cursos hayan participado directivos, técnicos, docentes, investigadores, personal de la Secretaría de Emergencia Nacional (organismo rector de la gestión del riesgo de desastre en el Paraguay) se constituyó en una oportunidad para que los participantes amplíen sus conocimientos y compartan sus experiencias en cuanto a la aplicación de la GRRD en sus instituciones.

\section{CONCLUSIONES}

Los documentos analizados permitieron: identificar las etapas y actividades desarrolladas en la ejecución del proyecto objeto de estudio y reconocer los resultados más resaltantes alcanzados según la percepción de los participantes, además de buenas prácticas que pueden ser replicadas en otras instituciones: la organización de talleres sobre temas relacionados con la GRRD, la evaluación de la seguridad de la institución, la convocatoria a actores de diferentes instituciones en las actividades de capacitación, la inclusión en el proyecto de acciones que afectan a la formación (talleres) e investigación (estudio y diagnóstico) como funciones de las IES.

Cabe aclarar que la principal limitación que este trabajo enfrentó fue la escasa información publicada acerca del proyecto y sus diferentes productos, situación que afectó la profundidad del análisis, por lo cual se recomienda la sistematización rigurosa del proyecto y la publicación en medios accesibles.

Declaración de interés. Los autores declaran no tener conflicto de intereses.

\section{REFERENCIAS BIBLIOGRÁFICAS}

Casulo, J. (2018). The Objectives of Sistainable Development and the academy Introducción.MEDISAN,22(8), 1089- 11 00. http://scielo.sld.cu/pdf/san/v22n8/1029-3019-san-2208-838.pdf

García-Lemus, V., Salguero, R., Maldonado, P. \& Gómez-Ruíz, Z. X. (2017). Guía de Evaluación del Índice de Seguridad en Instalaciones Universitarias-ISIU. Guatemala.

INAES. (2019). Proyecto ISE. "Hacia una Institución segura y resiliente : campaña universidades sostenibles y resilientes. Asunción. Red Universitaria de las Américas y el Caribe para la reducción de riesgo de desastres. https://www.inaes.edu.py/application/files/1715 /7659/4542/Libro_ISE_2020_ultima_version.pdf

Narváez, L., Lavell, A. \& Pérez Ortega, G. (2009). La Gestión del riesgo de desastres: un enfoque basado en procesos, p. 33 . www.comunidadandina.org

Peraldo, G. \& Acevedo, B. (2014). El reflejo de la Gestión del Riesgo en las páginas de la Revista Geológica de América Central. Revista Geológica de América Central, November. https://doi.org/10.15517/rgac.v0i0.16573

REDULAC. (2018). Campaña universidades sostenibles y resilientes. Asunción. Red Universitaria de las Américas y el Caribe para la reducción de riesgo de desastre.

Resolución aprobada por la asamblea general el 3 de Junio de 2015 (Naciones Unidas).http://www2.ohchr.org/spanish/b odies/hrcouncil/docs/gaA.RES.60.1_Sp.pdf

Reyes-Rivero, A. C., Montilla Pacheco, A. D. J., Castillo Garcia, P. G. \& Zambrano Vera, M. N. (2017). Amenaza, vulnerabilidad y riesgo ante eventos naturales. Factores socialmente construidos. Journal of Science and Research: Revista Ciencia e Investigación, 2(6), 22-28. https://doi.org/10.26910/issn.25288083 vol2iss6.2017pp22-28

UNISDR. (2015). Marco de Sendai para la Reducción del Riesgo de Desastres 2015-2030. https://www.unisdr.org/files/43291_spanishsendaiframew orkfordisasterri.pdf 